\title{
A diagnostic checklist for generalized dermatitis
}

This article was published in the following Dove Press journal:

Clinical, Cosmetic and Investigational Dermatology

\section{Susan Nedorost ${ }^{1,2}$ \\ 'Department of Dermatology, University Hospitals Cleveland Medical Center, Cleveland, $\mathrm{OH} 44106$. USA; ${ }^{2}$ Department of Population and Quantitative Health Sciences, Case Western Reserve University, Cleveland, OH 44I06 USA}

Correspondence: Susan Nedorost Department of Dermatology, University Hospitals Cleveland Medical Center, Case Western Reserve University, II 100 Euclid Avenue, Cleveland, $\mathrm{OH} 44 \mathrm{I06}$, USA

Tel +l 2168443872

$\mathrm{Fax}+\mathrm{I} 2168448993$

Email stn@case.edu
Purpose: Generalized dermatitis (defined as histological spongiotic dermatitis affecting more than three anatomical areas of the skin surface) has many potential causes that mimic atopic dermatitis and contact dermatitis. If a treatable cause is missed, the patient may be treated with chronic immunosuppressive therapy that carries more risk than specific treatment for a disease mimicking dermatitis. Checklists have been shown to improve patient safety, primarily in procedural contexts. This work assessed the utility of a diagnostic checklist for subacute and chronic generalized dermatitis in patients who had not improved after at least 1 month of avoidance of contact allergens identified by comprehensive patch testing, if indicated.

Patients and methods: Designed as a quality improvement project using Standards for Quality Improvement Reporting Excellence (SQUIRE) guidelines, a diagnostic checklist was used by the principal investigator for 1 year in a tertiary referral dermatitis clinic for patients without a confirmed cause for the dermatitis after two to three visits with the investigator. All patients had had diagnostic patch testing if indicated. Almost all had undergone skin biopsy by their referring provider. Fifteen patients met the criteria for inclusion in this study. Outcome measures included provider and patient perception of efficiency and/or confusion caused by the checklist. Length of time from the initiation of use of the checklist to final diagnosis was recorded. Additional diagnoses considered that were not included in the initial checklist were added to the checklist during the course of the study.

Results: The checklist was useful in improving diagnostic efficiency (prompting consideration of diagnoses not otherwise considered upon initiation of the visit that resulted in a final plan of care) in these complex cases of recalcitrant dermatitis. Open utilization of the checklist by the investigator during the clinical encounter was well accepted by patients and families.

Conclusion: Checklists can be useful for complex cognitive diagnostic work.

IRB approval status: University Hospitals Cleveland Medical Center Institutional Review Board \# 11-15-34.

Keywords: diagnostic error, patient safety, negative or irrelevant patch test results

\section{Introduction}

Dermatologists often encounter patients with subacute or chronic, generalized dermatitis, a multifactorial medical condition with a complex differential diagnosis including autoimmune, infectious, and malignant diseases.

Checklists have improved safety in health care and other industries. ${ }^{1}$ Checklists are more commonly used for procedural tasks rather than cognitive tasks. This quality improvement project examined a checklist for the cognitive task of diagnosis of patients with generalized dermatitis for whom 1 month of avoidance of allergens identified with comprehensive patch testing did not result in improvement. 
Dermatitis, especially when generalized over a large area of the skin, is a major burden. ${ }^{2}$ Generalized dermatitis often induces prolonged sleep deprivation and impaired ability to concentrate with significant effects on the quality of life. In turn, difficulty concentrating impedes communication during the brief time allotted for physician office visits. Patients are often focused on the immediate relief of symptoms; however, effective immediate relief requires use of systemic immunosuppressive medications, which hinders the diagnostic work-up in the short term and has significant health risks if used long term.

The impact of severe itch often leads patients to accept immunosuppressive therapy even knowing that it may shorten their life span. In some patients with recalcitrant dermatitis, identification of a culprit contact allergen (eg, avoidable by changing the brand of shampoo) may be a steroid-sparing intervention and eliminate the need for long-term immunosuppressive therapy. A complete work-up for underlying cause(s) of the dermatitis should be considered as a medical necessity in recalcitrant dermatitis. There is a complex juxtaposition between the patient's desire for immediate symptomatic relief and the physician's desire to minimize long-term risk to the patient by therapeutic interventions.

There is no widely accepted algorithm for the evaluation of patients with generalized dermatitis, especially those who have had comprehensive patch testing without identification of a relevant allergen. ${ }^{3}$ Furthermore, diagnostic tools such as comprehensive patch testing are not always accessible. Therefore, many patients are treated with chronic immunosuppression (eg, prednisone, methotrexate, and mycophenolate mofetil) without completing the diagnostic elements on the checklist. This puts them at unnecessary risk for serious infection and malignancy due to iatrogenic immunosuppression.

We lack data about how often this work-up is completed, as there is no way to detect how many patients treated with longterm immunosuppressives for generalized dermatitis might have been treated differently with a more precise diagnosis.

Dermatitis is often multifactorial, which further complicates the diagnostic work-up. Barrier dysfunction, allergen avoidance, and abnormalities in the microbiome may need to be addressed simultaneously in order to alleviate symptoms. A checklist serves as a reminder that more than one diagnostic or therapeutic element may be required.

In some cases, generalized itch without a primary rash can provoke so much scratching that dermatitis appears to be present, even though the itch is due to a systemic, noncutaneous cause. For this reason, systemic causes of itch are also in the differential diagnosis.
A diagnostic checklist can enhance patient safety by assuring patients and providers that no element of the diagnostic work-up has been omitted. Many of these diagnoses can be treated, or even cured, with interventions much less risky than chronic immunosuppressive therapy. The checklist includes a reminder to help patients understand the potential length of the diagnostic work-up in the course of evaluation and symptom-directed treatment.

\section{Methods}

Using Standards for Quality Improvement Reporting Excellence (SQUIRE) ${ }^{4,5}$ guidelines for a quality improvement project, a survey was designed for physicians evaluating patients with generalized dermatitis. The survey inquired about physician's perception of the efficiency (defined as time and resources needed to reach a final care plan) of the diagnostic checklist for generalized dermatitis. The survey also asked if the physician shared the checklist with patients, whether this enhanced or decreased patient engagement, and whether the checklist caused any confusion or detracted from clinical care.

For each patient, the length of the diagnostic work-up from the time of the physician's initial encounter with the patient, the number of diagnostic items excluded, and the final diagnosis were also queried. Any diagnoses considered that were not included in the checklist were added.

Checklist items could be completed in any order. The checklist includes diagnostic items for allergic contact dermatitis, diagnostic/therapeutic trials aimed at microbiome factors in dermatitis, and diagnostic tests for mimics of dermatitis. Tips for increasing the accuracy of these tests were included (eg, screening tests for hypereosinophilic syndrome should be conducted off of systemic steroids and multiple serological and tissue tests may be needed to diagnose urticarial phase bullous pemphigoid, where direct immunofluorescence may be less sensitive than in bullous phase disease). ${ }^{6}$

Information without protected health information identifiers was obtained for the patients evaluated with the checklist including age range, gender, and membership in a underrepresented minority or socially disadvantaged group (without further specification).

\section{Results}

The author used the checklist in a tertiary referral Dermatitis Clinic in the Midwest in 2016-2017. The patients in this clinic were referred for the consideration of patch testing. The checklist was used only for patients without a diagnosis after 2-4 clinical visits. During the study interval, over 300 
patients were referred and 106 patients received comprehensive patch test evaluation in this tertiary care clinic. The checklist was not used for patients with a diagnosis established at the initial consultation visit or the first two follow-up visits.

Because a majority of the patients $(95 \%)$ received a diagnosis either at the consultation visit or at the following 2-3 follow-up visits, the checklist was used for only 15 patients. Fourteen (93\%) patients had undergone patch testing, and one patient was assessed as not likely to benefit from patch testing. Patients who were not improved at the post-patch testing visit were included and four ultimately had a final diagnosis of systemic contact dermatitis or systemic contact dermatitis in conjunction with another condition.

Additional diagnostic considerations were added during the 1 year implementation process, and the format was refined (Table 1). The order of tests in the checklist reflects the frequency of use during the study interval.

Older and white patients were overrepresented in this cohort compared to the population of the county from which

Table I Final checklist informed by study results

\begin{tabular}{|c|c|c|}
\hline Check & Diagnosis/action to consider & Comment/information if action is indicated \\
\hline & Framing communication & $\begin{array}{l}\text { There is no treatment for the itch of dermatitis that does not have } \\
\text { potentially serious side effects when used over the long term. It may take } \\
\text { many weeks to complete all of the work-up }\end{array}$ \\
\hline & Patch test for allergic contact dermatitis & $\begin{array}{l}\text { Patch test to include the personal and occupational contactants that are } \\
\text { known sensitizers, which are currently in the patients' environment; } \\
\text { photo-patch testing may be needed to properly evaluate some } \\
\text { sunscreens, plants, and fragrances }\end{array}$ \\
\hline & $\begin{array}{l}\text { Complete blood count with differential for } \\
\text { hypereosinophilia }\end{array}$ & $\begin{array}{l}\text { Perform test when the patient is off systemic corticosteroids; other } \\
\text { useful studies: vitamin BI2 and tryptase (in combination); flow cytometry, } \\
\text { cytogenetics, and fluorescent in-situ hybridization studies if needed }\end{array}$ \\
\hline & Tests for (non)bullous pemphigoid & $\begin{array}{l}\text { Nonbullous (urticarial-phase) pemphigoid often requires direct } \\
\text { immunofluorescence, indirect immunofluorescence, and bullous } \\
\text { pemphigoid antigens by ELISA for diagnosis }\end{array}$ \\
\hline & Microscopic examination of scraping for scabies & Mineral oil preparation for patients with facial sparing \\
\hline & $\begin{array}{l}\text { Inquire about the use of exposure to hot tub (contact } \\
\text { allergy to "shock" treatment chemicals) }\end{array}$ & In patients with facial sparing ${ }^{7}$ \\
\hline & Inquire about exposure to sources of mites & $\begin{array}{l}\text { Discrete very pruritic lesions present, ask about pets (cheyletiella), } \\
\text { contact with birds, farming, cheese making, and oak trees }{ }^{8}\end{array}$ \\
\hline & $\begin{array}{l}\text { Examine for signs of (often amyopathic) } \\
\text { dermatomyositis }\end{array}$ & Consider myositis panel \\
\hline & Eczematous drug eruption & $\begin{array}{l}\text { Review medications for temporal association, discontinue prescription } \\
\text { and over-the-counter medicines if potential association with drug rash, } \\
\text { and evaluate for improvement. Patch testing may be performed for } \\
\text { eczematous and fixed drug reaction }\end{array}$ \\
\hline & $\begin{array}{l}\text { Inquire about exposure to hydroxyethyl starch as a } \\
\text { volume expander,' recreational drugs, or history of } \\
\text { biliary tract obstruction }\end{array}$ & $\begin{array}{l}\text { Rash appears to be secondary to scratching and no primary lesions on } \\
\text { examination }\end{array}$ \\
\hline & Additional biopsies rule out cutaneous $\mathrm{T}$ cell lymphoma & Accentuation of dermatitis in photoprotected skin \\
\hline & Test for dermatographism & Rash is accentuated by tight contact with garments or pressure \\
\hline & $\begin{array}{l}\text { Trial of gabapentin (rule out multilevel symmetric } \\
\text { neuropathic pruritus) }\end{array}$ & $\begin{array}{l}\text { Dermatitis is fixed in distribution, in patients with risk factors for } \\
\text { degenerative disk disease }{ }^{10}\end{array}$ \\
\hline & $\begin{array}{l}\text { Empiric treatment with systemic azole, followed } \\
\text { by gentle acidification with cleansers and a topical } \\
\text { azole body wash maintenance for the treatment of } \\
\text { seborrheic dermatitis component }\end{array}$ & Postpubertal patients with head and upper torso predominance \\
\hline & $\begin{array}{l}\text { Bacterial culture of skin to select antistaphylococcal } \\
\text { antibiotics }\end{array}$ & $\begin{array}{l}\text { Patients with the history of childhood onset "eczema"; necessary to } \\
\text { follow bleach bath with rinsing; and low } \mathrm{pH} \text { products to counteract } \\
\text { irritancy }\end{array}$ \\
\hline & Bone marrow biopsy & Patients with suspected myelofibrosis \\
\hline & $\begin{array}{l}\text { Assess for lower extremity edema provoking auto } \\
\text { eczematization }\end{array}$ & $\begin{array}{l}\text { Patients with stasis dermatitis; discontinue amlodipine if a current medication; } \\
\text { compression in conjunction with calf-pumping exercises }\end{array}$ \\
\hline & Obtain history of travel to Southwest US & $\begin{array}{l}\text { Coccidiomycosis, generalized hypersensitivity type can rarely present as } \\
\text { dermatitis }\end{array}$ \\
\hline & Inquire about implanted device & Temporal association and anatomical accentuation \\
\hline & Consider paraneoplastic syndrome & For example, Bazex if acral accentuation \\
\hline
\end{tabular}

Note: Italicized verbiage was added during the initial usage interval of this study. 
our hospital draws most patients where only $17 \%$ of the population is over 65 years. Three of the $15(20 \%)$ subjects were underrepresented minorities compared to approximately $33 \%$ of the county residents.

Time from first visit to final completion of the checklist was more than 20 weeks for eight of the 15 (53\%) patients, 13-20 weeks for three (20\%) patients, 9-12 weeks for three (20\%) patients, and less than 2 weeks for only one patient. This does not include time of the diagnostic work-up by referring providers; in most cases, the patients had seen several providers for the same symptoms prior to coming to this clinic. Examples of reasons for the time elapsed included 1 month avoidance trials to assess the relevance of allergens producing positive patch tests, systemic medication avoidance trials, time needed to receive results of ELISA for bullous pemphigoid antigens and indirect immunofluorescence for antibodies, and tapering interval to discontinue prednisone to allow an accurate measurement of peripheral eosinophils. Many patients had intermittent treatment with prednisone, and less commonly cyclosporine, to alleviate symptoms while awaiting test results. All patients had used multiple topical medications and emollients without benefit.

Eight of the 15 surveys indicated the checklist made the diagnostic work-up more efficient for the investigating clinician, as the checklist prompted evaluation for a diagnosis not readily considered when initiating the clinical encounter. Ten patients were shown the survey during their clinical visit, and in six of these cases, the investigating physician perceived that this enhanced patient engagement. In no case did sharing the checklist appear to increase patient anxiety.

In at least two cases, the checklist led to definitive diagnosis with first use. One of these was a patient with scabies where the referring provider had already treated for scabies, and the author was not prompted to reconsider the diagnosis of inadequately treated scabies until using the checklist. The other was a patient with bird mite sensitivity, where the primary occupation did not involve bird exposure, and the author had not elicited the exposure history until using the checklist prompted the specific question.

Five patients did not have a definitive diagnosis at the time the survey was complete:

- One had resolution of symptoms with self-prescribed bleach baths despite not having a history of childhood onset; this patient presented with a polymorphic dermatitis with psoriasiform features.

- One had a history of systemic contact dermatitis to benzoic acid and propylene glycol previously controlled with avoidance but after 2 years relapsed despite avoidance in food and medication; this patient had partial benefit with gabapentin treatment for suspected multisegmental neuropathic pruritus.

- One was treated with mycophenolate mofetil for suspected drug eruption to cardiac medications that could not be discontinued.

- One was treated with methotrexate for "dermal hypersensitivity" by co-managing rheumatologist for what may have been rash due to a biological medication that was not discontinued.

- One patient was not given a specific diagnosis, following completion of the checklist, atopy patch testing, trial of bleach and vinegar baths for possible atopic dermatitis, consultation by another dermatitis expert and a rheumatologist, and failure to respond to several empiric treatments including prednisone, azathioprine, gabapentin, and mirtazapine.

The investigator noted that it is difficult to entirely rule out several diagnoses that may have nonspecific histology. Cutaneous T-cell lymphoma could not be excluded in some patients. Amyopathic dermatomyositis can present as an eczematous rash without classic histology. Multilevel symmetric neuropathic pruritus is best diagnosed with a diagnostic and therapeutic trial of gabapentin.

The following diagnoses not on the initial checklist were considered, but none were confirmed, in this cohort: Bazex syndrome, coccidiomycosis, central nervous system disorder, myelofibrosis, and reaction to implanted device. The clinical investigator diagnosed several cases of stasis dermatitis with autoeczematization during the study interval in initial visits such that the checklist was never used for these patients, which prompted this diagnosis to be added to the final version of the checklist.

\section{Discussion}

Use of a diagnostic checklist for cognitive tasks is uncommon in dermatologic practice but was useful from both the provider and patient perspectives in this study.

The checklist includes a reminder to discuss the possibility of a long diagnostic work-up with patients; earlier use of the checklist would likely result in more realistic expectations for patients regarding the length of the diagnostic work-up. Utilizing the checklist only for patients, where diagnostic attempts by the referring providers (often more than three of whom were dermatologists) and after initial additional diagnostic work-up by this investigator were inconclusive, created a scenario where the checklist was useful to alleviate, but not to prevent, patient 
frustration. An unmet need is better understanding from the patient's perspective of how to best reduce risk and alleviate suffering during the diagnostic work-up of dermatitis. Shared decision making regarding temporary use of systemic medications during the diagnostic work-up interval is important.

Because patients in this tertiary clinic were referred for the consideration of patch testing and had received previsit patient education on patch testing, this was the first test utilized in many cases and patients diagnosed with ACD were therefore not included in this cohort. Therefore, this quality improvement project cannot demonstrate the diagnostic utility of patch testing in this highly selected patient cohort.

\section{Limitations}

Validation of the diagnostic checklist in other patient cohorts is warranted. Consideration should be given to adding additional diagnoses that are considered or confirmed in those cohorts. None of the patients in this checklist study had regular flares of dermatitis since childhood, which the investigator considers necessary for a diagnosis of atopic dermatitis. Referral bias was likely given the racial imbalance compared to the county in which our medical center is located.

\section{Conclusion}

The data obtained suggest that

1. the checklist should be used early in the diagnostic workup so that patients understand the potential length and complexity of the work-up;

2. use of the checklist reassures patients when they are anxious about the lack of a diagnosis during the work-up;

3. the checklist may remind providers to consider an unusual or common (but undertreated) diagnosis and to be sure that all diagnostic tests were conducted under valid conditions; and

4. the checklist reminds providers of tasks that should be completed before beginning immunosuppressive treatments as a last resort, to alleviate suffering for patients where a definitive diagnosis cannot be identified.

The checklist is not intended to replace, but rather to augment, careful history and physical examination and clinical reasoning.

\section{Disclosure}

The author reports no conflicts of interest in this work.

\section{References}

1. Gawande A. The Checklist Manifesto: How to get Things Right. New York: Metropolitan Books; 2010.

2. Karimkhani C, Dellavalle RP, Coffeng LE, et al. Global Skin Disease Morbidity and Mortality: An Update From the Global Burden of Disease Study 2013. JAMA Dermatol. 2017;153(5):406-412.

3. Spiker A, Mowad C. Patch Test Negative Generalized Dermatitis. Dermatitis. 2016;27(5):259-262.

4. SQUIRE 2.0 [webpage on the internet] updated 2017. Available from: http://www.squire-statement.org/index.cfm? fuseaction=page. viewpage\&pageid=471. Accessed October 4, 2018.

5. Ogrinc G, Davies L, Goodman D, Batalden P, Davidoff F, Stevens D. SQUIRE 2.0 (Standards for QUality Improvement Reporting Excellence): revised publication guidelines from a detailed consensus process. BMJ Qual Saf. 2016;25(12):986-992.

6. Elston DM, Stratman EJ, Miller SJ. Skin biopsy: Biopsy issues in specific diseases. J Am Acad Dermatol. 2016;74(1):1-16.

7. Kagen MH, Wolf J, Scheman A, Nedorost S. Potassium peroxymonosulfate-induced contact dermatitis. Contact Dermatitis. 2004;51(2):89-90.

8. Sceppa JA, Lee YH, Jacobs SB, Adams DR. What's eating you? Oak leaf itch mite (Pyemotes herfsi). Cutis. 2011;88(3):114-116.

9. Ständer S, Richter L, Osada N, Metze D. Hydroxyethyl starch-induced pruritus: clinical characteristics and influence of dose, molecular weight and substitution. Acta Derm Venereol. 2014;94(3):282-287.

10. Ward RE, Veerula VL, Ezra N, Travers JB, Mousdicas N. Multilevel symmetric neuropathic pruritus (MSNP) presenting as recalcitrant “generalized” pruritus. J Am Acad Dermatol. 2016;75(4):774-781.
Clinical, Cosmetic and Investigational Dermatology

\section{Publish your work in this journal}

Clinical, Cosmetic and Investigational Dermatology is an international, peer-reviewed, open access, online journal that focuses on the latest clinical and experimental research in all aspects of skin disease and cosmetic interventions. This journal is included on PubMed. The manuscript management system is completely online
Dovepress

and includes a very quick and fair peer-review system, which is all easy to use. Visit http://www.dovepress.com/testimonials.php to read real quotes from published authors 